\title{
Model of white spot syndrome virus (WSSV) epidemics in Litopenaeus vannamei
}

\author{
Jeffrey M. Lotz*, M. Andres Soto** \\ Department of Coastal Sciences, University of Southern Mississippi, Gulf Coast Research Laboratory, PO Box 7000 , \\ Ocean Springs, Mississippi 39566-7000, USA
}

\begin{abstract}
White spot syndrome virus (WSSV) is devastating shrimp aquaculture throughout the world, but despite its economic importance no work has been done on modeling epidemics of this pathogen. Therefore we developed a Reed-Frost epidemic model for WSSV in Litopenaeus vannamei. The model includes uninfected susceptible, latently infected, acutely infected, and dead infected shrimp. The source of new infections during an outbreak is considered to be dead infected shrimp. The transmission coefficient, patency coefficient, virulence coefficient, and removal coefficient (disappearance of dead infected shrimp) control the dynamics of the model. In addition, an explicit area parameter is included to help to clarify the distinction between density and absolute shrimp population size. An analysis of the model finds that as number of shrimp, initial dose, transmission coefficient, patency coefficient, virulence coefficient, or removal coefficient changes, the speed of the epidemic changes. The model predicts that a threshold density of susceptible shrimp exists below which an outbreak of WSSV will not occur. Only initial dose, transmission coefficient, removal coefficient, and area coefficient affect the predicted threshold density. Increases in the transmission coefficient reduce the threshold value, whereas increases in the other factors cause the threshold value to increase. Epidemic models may prove useful to the shrimp aquaculture industry by suggesting testable hypotheses, some of which may contribute to the eventual control of WSSV outbreaks.
\end{abstract}

KEY WORDS: WSSV · Epidemic model · White spot syndrome virus · Epidemiology $\cdot$ Shrimp disease

\section{INTRODUCTION}

White spot syndrome virus (WSSV), a pernicious pathogen of marine shrimp, is ravaging shrimp farms throughout the world. WSSV is a rather large bacilliform virus, formerly referred to the nonoccluded baculoviruses but presently unassigned by the International Committee on Taxonomy of Viruses (Murphy et al. 1995). It was recognized in the early 1990s in eastern and southeastern Asia, but over the past several

\footnotetext{
*E-mail: jeff.lotz@usm.edu

** Present address: Biology Department, Texas A+M University-Kingsville, 700 University Blud., Kingsville, Texas 78363, USA
}

years has spread rapidly, and its distribution has become almost coextensive with the industrial cultivation of penaeid shrimp. WSSV is currently the major shrimp pathogen affecting the culture of shrimp in both the eastern and western hemispheres.

The progression of a WSSV infection in affected ponds begins when shrimp become anorexic, and in 1 to $2 \mathrm{~d}$ mass mortalities ensue. By 3 to $10 \mathrm{~d}$ following onset of mortalities, deaths typically reach proportions greater than 0.80, and frequently all the population succumbs (Nakano et al. 1994, Lightner 1996). In laboratory exposures, mortalities begin 24 to $48 \mathrm{~h}$ postexposure, and complete mortalities are typically achieved after 2 to 12 d (Chou et al. 1995, Lightner et al. 1998, and our Fig. 1). There are few survivors of WSSV in laboratory challenges, although live penaeid 


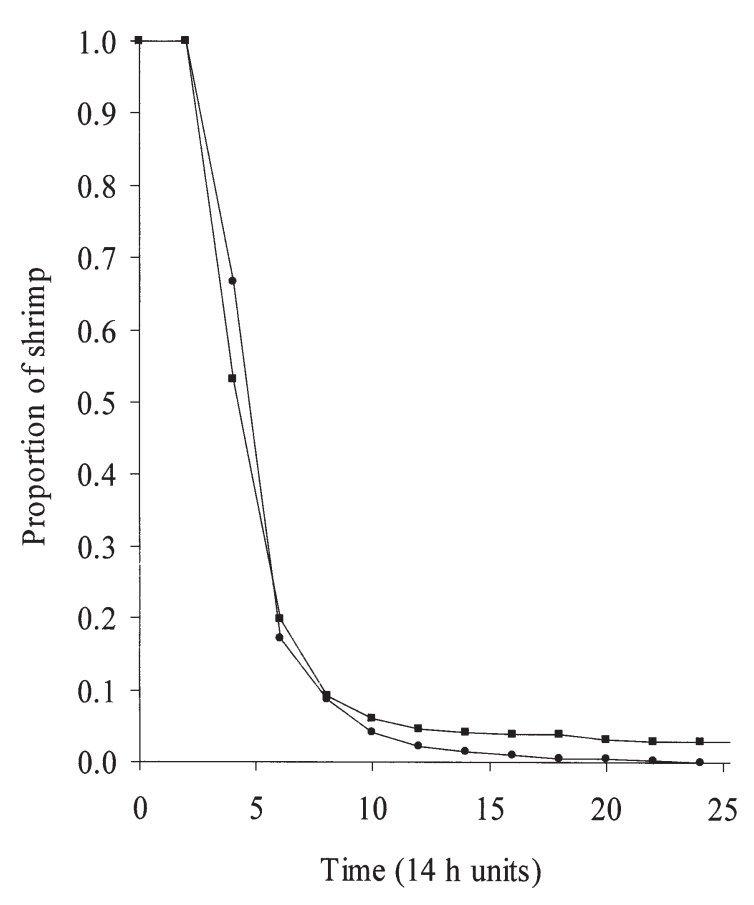

Fig. 1. Litopenaeus vannamei. Illustration of typical experimental survival curves of $3 \mathrm{~g}$ shrimp exposed per os to a single dose ( $3 \%$ of body weight) of WSSV-infected shrimp cephalothoraces. Final proportion survivals were 0.029 and 0.00. Number of shrimp in each exposure was 700 and area of each exposure tanks was $4 \mathrm{~m}^{2}$

shrimp with WSSV have been reported from wild catches and from affected ponds (Lo et al. 1996, Flegel 1997, Tsai et al. 1999).

Despite general descriptions of the course of a WSSV epidemic in populations of shrimp (Chou et al. 1995, Lightner 1996, Lightner et al. 1998), there is no systematic framework to address questions that are important for understanding the epidemiology of infections in wild and cultured populations of shrimp. The eventual goal of shrimp viral epidemiology is to understand and to control the dynamics of serious viral pathogens, and epidemic models can facilitate the goal. In this contribution we present a preliminary model of WSSV epidemics in the penaeid shrimp Litopenaeus vannamei. First, a diagrammatic representation of a generalized WSSV epidemic is presented to illustrate important components and connections among those components. We then transform the schematic into a mathematical model of the epidemic, and apply laboratory estimates of the important coefficients to gain some understanding of model WSSV epidemics in L. vannamei. Finally, using the model we generate some hypotheses about factors that might affect the course of an epidemic of WSSV in L. vannamei and might lead to successful management strategies.

\section{MODELLING PROCEDURE}

Diagrammatic representation of a WSSV epidemic. A WSSV infection in a shrimp can be divided into several states. The initial state is a short asymptomatic latent period during which the virus multiplies, eventually causing a symptomatic acute infection. The acute infections may progress to cause death of the shrimp or, possibly, acutely infected shrimp survive with chronic infections or even recover completely from infection.

Translating the course of infection in an individual shrimp into the dynamics of infection in a population of shrimp requires understanding the different states of infection, the transition probabilities between states, and the sources of infection. In our model of a WSSV epidemic, 6 host states are identified: (1) susceptible hosts are liable to infection, (2) latent hosts carry latent infections, (3) acute hosts carry acute infections, (4) dead hosts are infected and have died from infection, (5) chronic hosts have recovered from acute infections and are carriers, and (6) removed hosts are dead infected hosts that are no longer infectious because they have been eaten or have decomposed (Fig. 2).

The arrows in Fig. 2, connecting one host state to another, map the course of infection in a population. The arrows connecting a different host state to the suscepti-

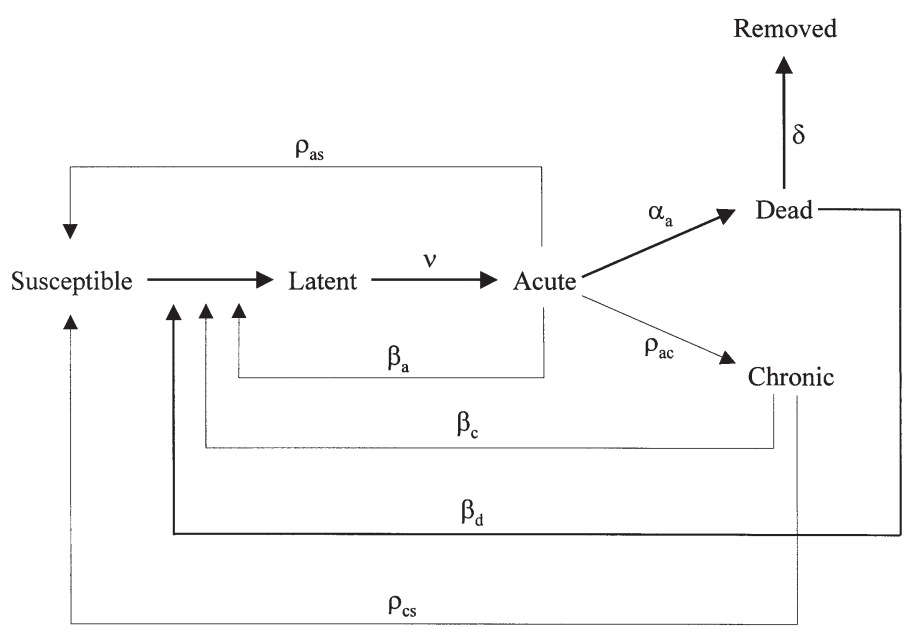

Fig. 2. Litopenaeus vannamei. Diagram of a hypothetical WSSV epidemic. $\beta_{\mathrm{d}}$ : transmission coefficient from a dead infected carcass to a susceptible shrimp; $\beta_{a}$ : transmission coefficient from an acutely infected shrimp; $\beta_{\mathrm{c}}$ : transmission coefficient from a chronically infected shrimp; v: patency coefficient; $\rho_{\text {as }}$ : coefficient of recovery of an acutely infected shrimp by elimination of the infection and becoming susceptible; $\rho_{\mathrm{ac}}$ : coefficient of recovery of an acutely infected shrimp by becoming a chronically infected survivor; $\rho_{\mathrm{cs}}$ : coefficient of recovery of a survivor with a chronic infection by elimination of infection to become susceptible to re-infection; $\alpha_{a}$ : coefficient of mortality of an acutely infected shrimp; $\delta$ : removal coefficient for dead infected shrimp losing infectivity 
ble-latent arrow identify the sources of infection. The various coefficients that appear on the arrows represent the probabilities of transition from one state to another during a unit of time. $\beta$ s represent the probabilities that a new infection occurs during one unit of time from an exposure between a susceptible and an infected host. $\beta_{\mathrm{d}}$ is the probability of transmission due to an exposure to a dead infected carcass, $\beta_{a}$ is the probability of transmission due to an exposure to an acutely infected shrimp, and $\beta_{\mathrm{c}}$ is the probability of transmission due to an exposure to a chronically infected shrimp. The patency coefficient $(v)$ is the probability that a latently infected shrimp develops a symptomatic or patent acute infection during 1 unit of time. The recovery coefficients are represented by the $\rho s . \rho_{\text {as }}$ is the probability that an acutely infected shrimp recovers from infection by eliminating the virus to become susceptible to infection again, $\rho_{\text {ac }}$ is the probability that an acutely infected shrimp recovers to become a chronically infected survivor, and $\rho_{\mathrm{cs}}$ is the probability that a chronically infected shrimp eliminates the virus to become susceptible to infection again. The virulence coefficient $\left(\alpha_{a}\right)$ is the probability of mortality in acutely infected shrimp (a measure of virulence), and $\delta$ is the removal coefficient or the probability that a dead infected shrimp loses its infectivity.

Shrimp can be infected from exposure to WSSV by injection of cell-free extract of infected tissue (Wongteerasupaya et al. 1995, Tapay et al. 1997), immersion in water containing cell-free extract of infected tissue (Chou et al. 1995, Wang et al. 1997), ingestion of infected tissue (Chang et al. 1996, Lightner et al. 1998), and cohabitation of susceptible shrimp with infected shrimp (Flegel et al. 1997, Soto \& Lotz 2001). However, not all modes of transmission, pathways or shrimp states are of equal importance during an epidemic.

Injection of shrimp with cell-free extract and immersion in water containing cell-free extract are standard laboratory methods for transmission but are of no importance as a natural mode of transmission during an epidemic. The normal routes of transmission in ponds and natural habitats are by way of cohabitation with infected shrimp that may be shedding the virus into the surrounding medium or ingestion of infected hosts. Work in our laboratory has demonstrated that cohabitation transmission $\left(\beta_{\mathrm{a}}\right)$ is over an order of magnitude lower than ingestion transmission $\left(\beta_{\mathrm{d}}\right)$ (Soto \& Lotz 2001). Therefore, we suggest that the most important route of transmission during a natural epidemic is via ingestion. We do not imply that cohabitation or waterborne transmission should be ignored - those mechanisms may be important for the introduction and geographic spread of the virus - we only imply that once an epidemic has started, the dominant route of transmission to uninfected shrimp is likely to be ingestion of dead infected carcasses (Soto \& Lotz 2001). For these reasons as well as for simplifying our model, we consider only ingestion transmission and (for now) ignore the other routes.

The presence of survivors, whether infected or not, are not considered in the model, because in laboratory challenges of Litopenaeus vannamei-which is the focus host of our model-there are few if any infected survivors (Lightner et al. 1998, Lotz unpubl. data and our Fig. 1). In addition, live shrimp are of lesser importance than dead shrimp for transmission, and omitting survivors simplifies the model.

In Fig. 2 the pathways represented by the lighter arrows are deemed less important epidemically than the pathways represented by the darker arrows. A simpler and more tractable representation of WSSV, focusing only on the most important pathways, is shown in Fig. 3.

Mathematical representation of a WSSV epidemic. The simplified diagram can be transformed into a set of mathematical equations that capture the essence of a WSSV epidemic:

$$
\begin{aligned}
S_{t+1} & =S_{t}-S_{t} \cdot\left(1-\left(1-\beta_{\mathrm{d}}\right)^{\frac{D_{t}}{\lambda}}\right. \\
L_{t+1} & =L_{t}+S_{t} \cdot\left(1-\left(1-\beta_{\mathrm{d}}\right)^{\frac{D_{t}}{\lambda}}-L_{t} \cdot v\right. \\
A_{t+1} & =A_{t}+L_{t} \cdot V-A_{t} \cdot \alpha_{\mathrm{a}} \\
D_{t+1} & =D_{t}+A_{t} \cdot \alpha_{\mathrm{a}}-D_{t} \cdot \delta
\end{aligned}
$$

where $S_{t}$ : shrimp susceptible to infection; $L_{t}$ : shrimp with latent infections; $A_{t}$ : shrimp with acute infections; $D_{t}$ : dead infections shrimp, at time $t$. The mathematical expression of the WSSV epidemic is a Reed-Frost model (Abbey 1952, Black \& Singer 1987). Eq. (1) represents the dynamics of $S$, where $\beta_{\mathrm{d}}$ is the transmission coefficient or the probability that exposure for 1 time unit to an infectious dead shrimp results in transfer of infection to the susceptible shrimp. The probability that 1 susceptible shrimp does not acquire an infection after exposure to every infectious shrimp is $\left(1-\beta_{\mathrm{d}}\right)^{D}$, and therefore the probability that a susceptible shrimp will become infected after exposure to all of the dead shrimp is $1-\left(1-\beta_{\mathrm{d}}\right)$.

Additionally, Eq. (1) contains coefficient $\lambda$ that accounts for the area a shrimp can traverse in 1 unit of time and allows for the possibility that shrimp are not

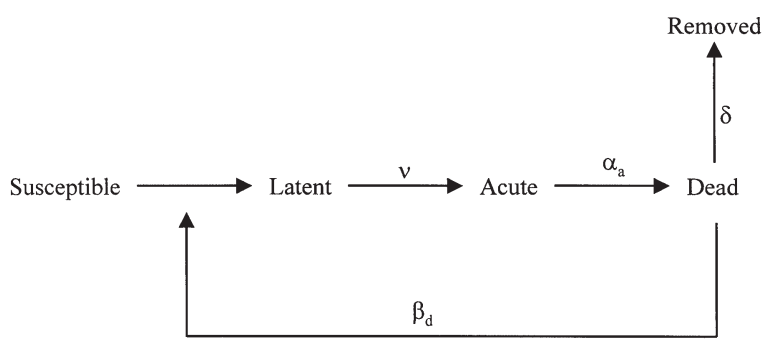

Fig. 3. Litopenaeus vannamei. Diagram of a simplified WSSV epidemic. Symbols as in Fig. 2 


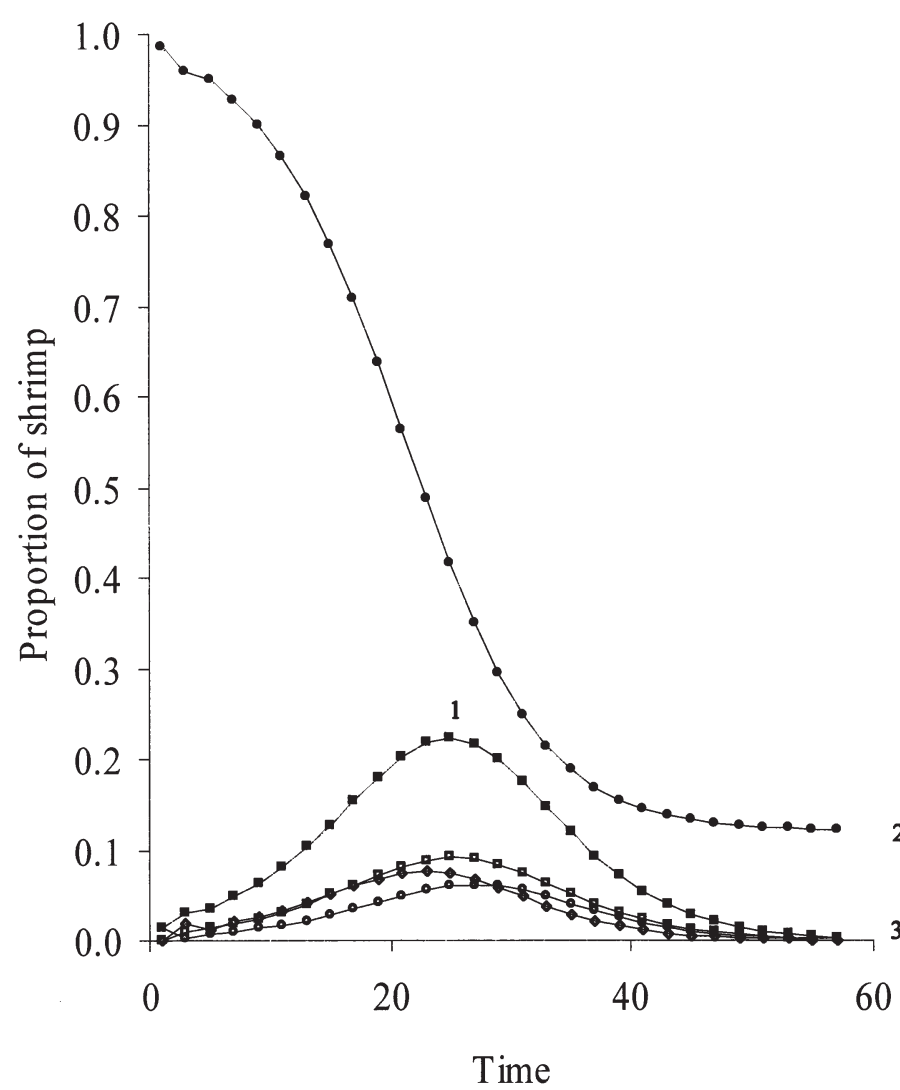

Fig. 4. Litopenaeus vannamei. Model epidemic illustrating characteristics of an epidemic. Parameter values were chosen to clearly display the epidemic characteristics. 1: epidemic peak at Time $25 ; 2$ : size of epidemic was 0.88 of susceptible shrimp infected; 3 : end of epidemic at Time 57. Transmission coefficient from a dead infected carcass, $\beta_{\mathrm{d}}=0.02$; patency coefficient, $v=0.5$; acute virulence coefficient, $\alpha_{a}=0.40$; removal coefficient of dead infectious carcasses, $\delta=0.60$; area coefficient, $\lambda=1$; initial number of susceptible shrimp, $S_{0}=70$; initial number of dead infected carcasses (dose), $D_{0}=1$. $(\bullet)$ susceptible shrimp; $(\diamond)$ latent infections; (ㅁ) acute infections; ( $($ ) dead infected shrimp; (ロ) total infections

randomly mixing in the entire area but only in some portion of the area. If a shrimp covers an area $k$ units large, e.g. $5 \mathrm{~m}^{2}$ in 1 unit of time and the total area of interest is $K$ units, say $50 \mathrm{~m}^{2}$, then the shrimp will cover $k / K$ or $(5 / 50=0.1)$ of the total area in a unit of time. The proportion of the total area that a shrimp can cover, $\mathrm{k} / \mathrm{K}$, can be reduced to $1 / \lambda$, and it follows that the proportion of possible exposures that can be made in a unit of time is also $1 / \lambda$, assuming that shrimp are homogeneously distributed in the area of interest. Ultimately we find that the total number of susceptible shrimp that become infected in 1 unit of time, each of which has been exposed to some proportion of the infectious shrimp is:

$$
S \cdot\left(1-\left(1-\beta_{\mathrm{d}}\right)^{\frac{D}{\lambda}}\right.
$$

Our use of $\lambda$ implies that the number of susceptible shrimp in the model is the absolute number rather than density. Density enters into the model separately because we include an explicit area coefficient. In our formulation, by manipulating either the area or the number of susceptible shrimp, the effect of density can be considered.

Eq. (2) represents the dynamics of $L$. In 1 unit of time the number of shrimp with latent infections increases by the number of susceptible shrimp that become infected and decreases by the number of shrimp with latent infections that develop into acute infections. The coefficient $v$ is the likelihood that a latent infection becomes acute in 1 unit of time.

Eq. (3) represents the dynamics of $A$. The number of shrimp with acute infections increases by the number of shrimp with latent infections that develop acute infections and decreases by the number of shrimp with acute infections that die.

Eq. (4) describes the dynamics of $D$ in the system. The number of dead shrimp increases as acutely infected shrimp die and decreases as the carcasses become noninfectious, either by being consumed or by decomposition. The probability that a dead infectious carcass becomes noninfectious in 1 unit of time is represented by $\delta$ (removal coefficient).

Characteristics of model epidemics. A display of the model epidemic described by Eqs. (1) to (4) features the epidemic curve, the epidemic peak, the duration of the epidemic, and the size of the epidemic (Fig. 4). Fig. 4 and Figs. 5 to 12 were generated by simulation using Mathcad 2000 software (Mathsoft). The values for the coefficients in Fig. 4 were selected to clearly illustrate the components of a model epidemic.

The epidemic curve graphs the number of infected shrimp during each time period of an epidemic (Bailey 1975, Daley \& Gani 1999). By convention, an epidemic occurs when there is a peaked epidemic curve (Bailey 1975) which is caused by an initial increase in infected individuals followed by an eventual ebb as the epidemic runs its course. For a WSSV epidemic, the curve includes all latent infections, acute infections, and infected dead (Fig. 4). Although we consider latent and acute infections to be of lesser importance in transmission than the infected dead, we include the latent and acute infections as part of the epidemic curve because they invariably become infected dead.

The epidemic peak (labelled 1 on Fig. 4) is the point at which the epidemic curve reaches its maximum (Bailey 1975), i.e. Time 25 on Fig. 4. A measure of the speed of an epidemic is the time necessary for an epidemic to reach its peak (Bailey 1975).

The epidemic duration is the width of the epidemic curve (Bailey 1975, Daley \& Gani 1999). In our model the epidemic duration is from the initial infection to the 
time the number of acute infections is less than 1 and the number of dead infected shrimp is less than 0.1 . We use 0.1 as a convention to acknowledge that a whole dead shrimp is not necessary to effect transmission. In Fig. 4 the epidemic duration is 57 time units.

Epidemic size is the proportion of susceptible shrimp that become infected during the epidemic (Bailey 1975; Daley \& Gani 1999). In Fig. 4, 0.88 of the susceptible shrimp have become infected when the infectious material disappears from the system.

The concept of epidemic threshold is an important outcome of the mathematical approach to epidemics. The threshold is the minimum number of susceptible individuals that allows an epidemic to occur (Kermack \& McKendrick 1927, Bailey 1975). By convention, we start our model epidemics with dead infected shrimp, and the threshold for our WSSV model is:

$$
\frac{-D_{0} \cdot \delta}{-1+\left(1-\beta_{d}\right)^{\frac{D_{0}}{\lambda}}}
$$

If the initial number of susceptible shrimp, $S_{0}$, is greater than Eq. (6), an epidemic (peaked epidemic curve) will ensue. If, however, $S_{0}$ is less than Eq. (6), a peak in the epidemic curve will not ensue and the pathogen will abate with no net increase in infectious material. Eq. (6) indicates that the threshold is a function of the relative rates of the disappearance of infectious shrimp and the appearance of new infectious material. If dead infected shrimp disappear faster than new infections are produced, an epidemic will not occur.

Characteristics of a theoretical WSSV epidemic in Litopenaeus vannamei. Fig. 5 illustrates a model epidemic of WSSV in L. vannamei resulting from coefficient values obtained in laboratory studies (Soto \& Lotz 2001, Soto et al. 2001, and our Table 1). The coefficient estimates were made for 12 shrimp in tanks with bottom areas of $1 \mathrm{~m}^{2}$, and exposed to 1 dead infected carcass. In this WSSV model epidemic, the epidemic peak

Table 1. Litopenaeus vannamei. Estimates of epidemiologic coefficients for WSSV. Estimates were obtained using 12 susceptible shrimp in a $1 \mathrm{~m}^{2}$ tank, for a time unit of $14 \mathrm{~h}$, and an initial dose of 1 infected cadaver

\begin{tabular}{|lcl|}
\hline Coefficient & Estimate (range) & Source \\
\hline Transmission $\left(\beta_{\mathrm{d}}\right)$ & $0.5(0.4-0.7)$ & $\begin{array}{l}\text { Soto \& Lotz (2001) } \\
\text { Soto et al. (2001) } \\
\text { Poto \& Lotz } \\
\text { (unpubl. data) }\end{array}$ \\
Virulence $\left(\alpha_{\mathrm{d}}\right)$ & $0.5(-)$ & $\begin{array}{l}\text { Soto \& Lotz (2001) } \\
\text { Soto et al. (2001) } \\
\text { Soto \& Lotz } \\
\text { (unpubl. data) }\end{array}$ \\
\hline
\end{tabular}

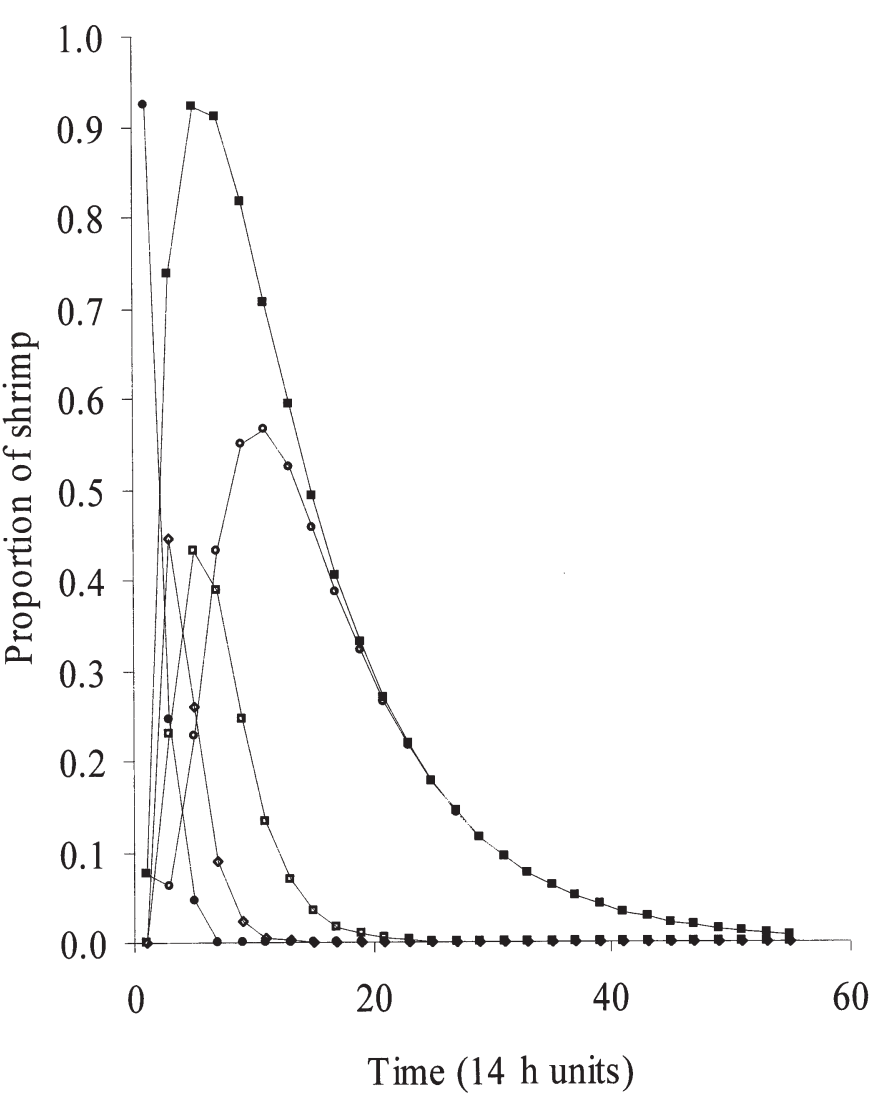

Fig. 5. Litopenaeus vannamei. Model WSSV epidemic. Coefficient values derived from laboratory estimates: epidemic peak at Time 3 ; size of epidemic: $100 \%$ of susceptible shrimp became infected; end of epidemic at Time 54. Transmission coefficient from a dead infected carcass, $\beta_{\mathrm{d}}=0.5$; patency coefficient, $v=0.5$; acute virulence coefficient, $\alpha_{a}=0.3$; removal coefficient of dead infectious carcasses, $\delta=0.1$; area coefficient, $\lambda=1$; initial number of susceptible shrimp, $S_{0}=12$; initial number of dead infected carcasses (dose), $D_{0}=1$. Symbols as in Fig. 4

occurs at Time 3, the end of the epidemic at Time 54, and the size of the epidemic is $100 \%$ (Fig. 5). The calculated threshold under these conditions is 0.2 susceptible shrimp $\mathrm{m}^{-2}$.

Effect of model conditions on characteristics of a WSSV epidemic in Litopenaeus vannamei. We studied the effects of changes in the initial number of susceptible shrimp, the initial dose of dead infected shrimp, and the epidemic coefficients on the characteristics of WSSV epidemics by simulating a series of model epidemics for each factor where only 1 factor was varied. Each factor affected epidemic size, time to epidemic peak, epidemic duration, and threshold of a model WSSV epidemic in L. vannamei in various ways (see Figs. 6 to 12). Table 2 summarizes the findings.

Initial number of susceptible shrimp $\left(\boldsymbol{S}_{\mathbf{0}}\right)$. To investigate the effect of different numbers of susceptible 


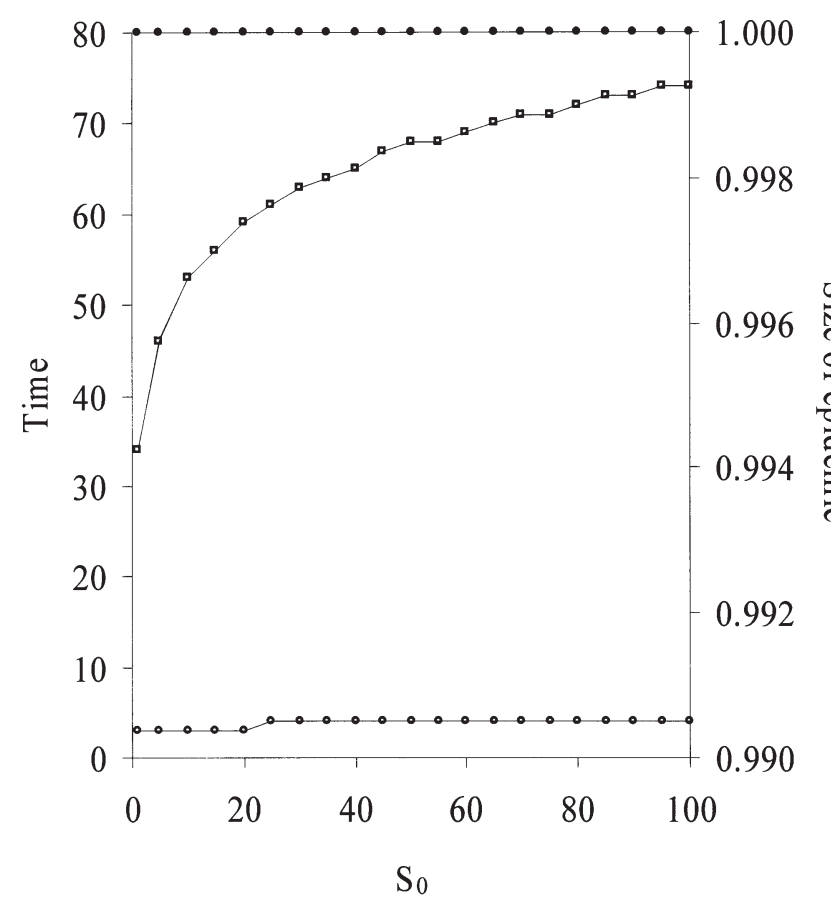

Fig. 6. Litopenaeus vannamei. Effect of number of shrimp $\left(S_{0}\right)$ on characteristics of model WSSV epidemics. Transmission coefficient from a dead infected carcass, $\beta_{\mathrm{d}}=0.5$; patency coefficient, $v=0.5$; acute virulence coefficient, $\alpha_{a}=0.3$; removal coefficient of dead infectious carcasses, $\delta=0.1$; area coefficient, $\lambda=1$; initial number of dead infected carcasses (dose), $D_{0}=1 .(\bullet)$ Epidemic sizes; $(\square)$ epidemic durations; ( $)$ times to epidemic peaks

shrimp on the characteristics of WSSV epidemics in Litopenaeus vannamei, we ran a series of simulations where $S_{0}$ was varied from 1 to 100 . The other conditions in the series of simulations were held at the values given in Table 1. Because the area coefficient was held constant during the simulations, not only was there a change in the number of susceptible shrimp but there was a concomitant change in the density of susceptible hosts. As the initial number of susceptible shrimp was increased, the duration and the time to peak of the epidemic increased. The final size of a WSSV epidemic in $L$. vannamei was unaffected by the initial number of shrimp and was 1.0 at all values (Fig. 6). Because the threshold was given in terms of the number of susceptible shrimp (Eq. 6), there could be no effect of the number of susceptible shrimp on the threshold value (Table 2).

Dose (initial infected dead $=\boldsymbol{D}_{\mathbf{0}}$ ). To investigate the effect of initial dose on the characteristics of model WSSV epidemics in Litopenaeus vannamei, we performed a series of simulations where the number of dead infected shrimp at the beginning of the exposure $\left(D_{0}\right)$ varied from 1 to 100 . The other conditions of the
Table 2. Relationship between model coefficients and epidemic characteristics. 0: no relationship; + : positive relationship; -: negative relationship; $S_{0}$ : initial number of susceptible shrimp; $D_{0}$ : initial number of dead infected carcasses

\begin{tabular}{|c|c|c|c|c|}
\hline \multirow[t]{2}{*}{ Model parameter } & \multicolumn{4}{|c|}{ Epidemic characteristic } \\
\hline & Threshold & $\begin{array}{c}\text { Time to } \\
\text { peak }\end{array}$ & Duration & Size \\
\hline \multirow[t]{2}{*}{$S_{0}$} & \multirow[t]{2}{*}{0} & + & + & \multirow[t]{2}{*}{+} \\
\hline & & $-{ }^{\mathrm{a}}$ & $-{ }^{a}$ & \\
\hline$D_{0}$ & + & - & + & $t^{a}$ \\
\hline Transmission $\left(\beta_{\mathrm{d}}\right)$ & - & _- & $\begin{array}{l}-^{\mathrm{a}} \\
-\end{array}$ & $t^{\mathrm{a}}$ \\
\hline Patency $(v)$ & 0 & - & - & $+^{\mathrm{a}}$ \\
\hline Virulence $\left(\alpha_{a}\right)$ & 0 & - & - & $+^{\mathrm{a}}$ \\
\hline Area $(\lambda)$ & + & + & + & - \\
\hline Removal ( $\delta)$ & + & + & - & $-{ }^{\mathrm{a}}$ \\
\hline \multicolumn{5}{|c|}{${ }^{\mathrm{a} F r o m ~ L o t z ~ e t ~ a l . ~(2001) ~}$} \\
\hline
\end{tabular}

epidemics were held constant at the values in Table 1. As $D_{0}$ increased, the duration of the epidemic increased but the time to peak was somewhat decreased (Fig. 7). The final size of the epidemic at all

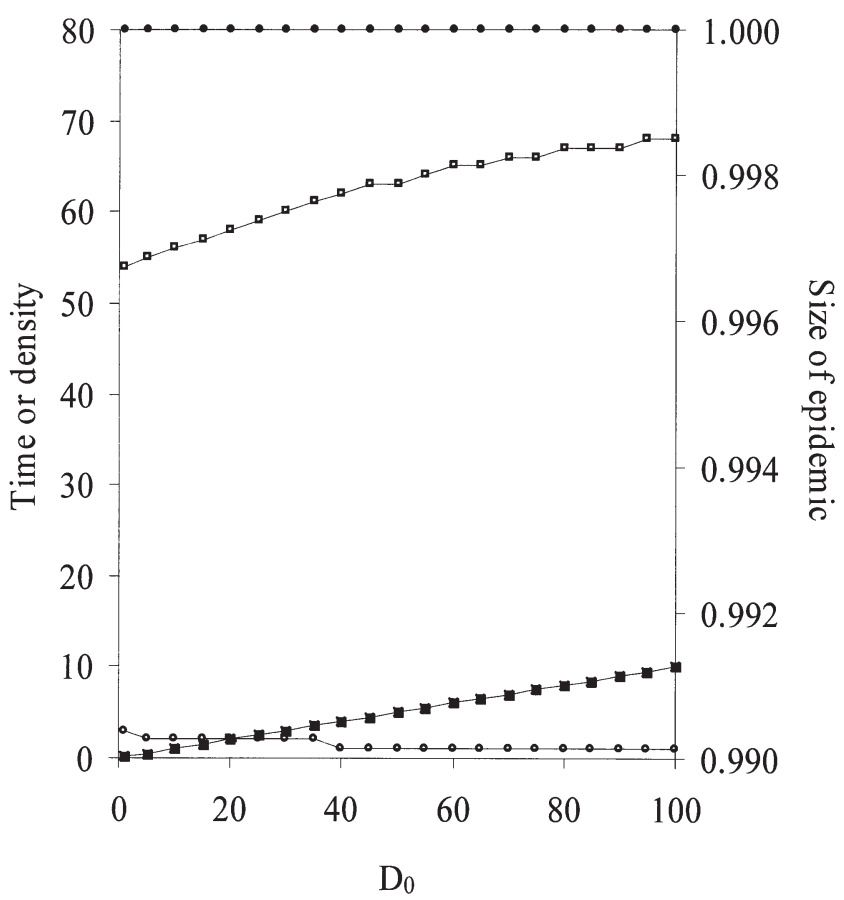

Fig. 7. Litopenaeus vannamei. Effect of initial number of dead infected carcasses (dose, $D_{0}$ ) on characteristics of a theoretical epidemic. Transmission coefficient from a dead infected carcass, $\beta_{\mathrm{d}}=0.5$; patency coefficient, $v=0.5$; acute virulence coefficient, $\alpha_{a}=0.3$; removal coefficient of dead infected carcasses, $\delta=0.1$; area coefficient, $\lambda=1$; initial number of susceptible shrimp, $S_{0}=12$. (匹) Epidemic threshold values; other symbols as in Fig. 6 


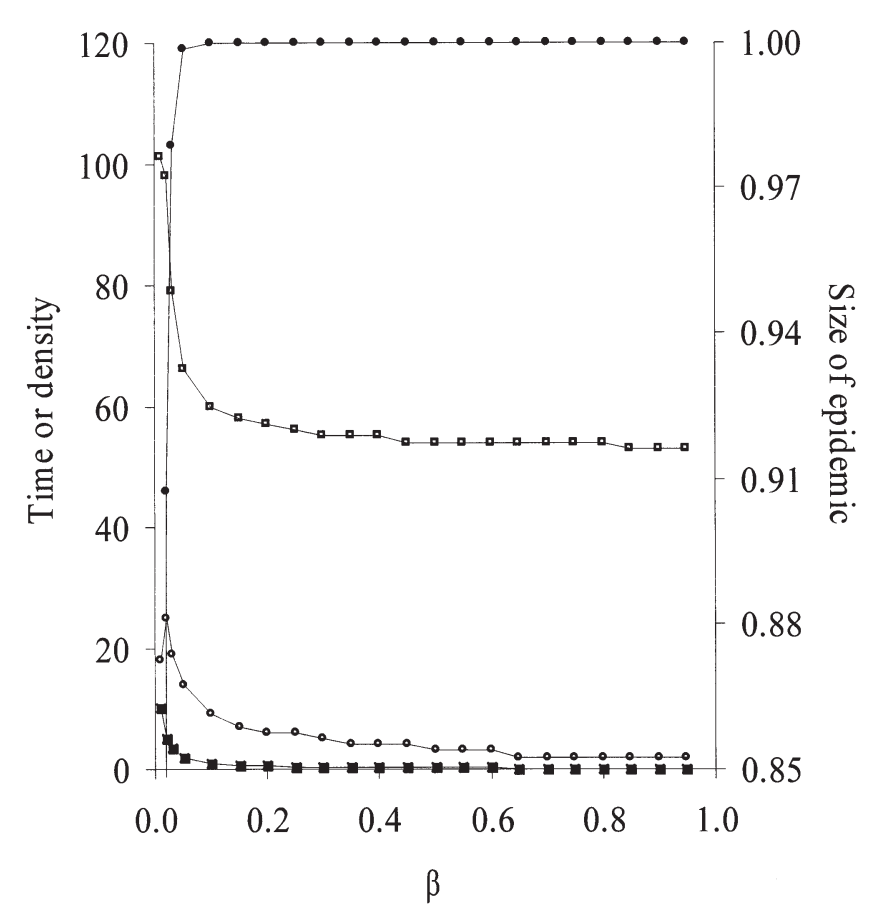

Fig. 8. Litopenaeus vannamei. Effect of transmission coefficient $\left(\beta_{\mathrm{d}}\right)$ on characteristics of model WSSV epidemics. Patency coefficient, $v=0.5$; acute virulence coefficient, $\alpha_{a}=0.3$; removal coefficient of dead infected carcasses, $\delta=0.1$; area coefficient, $\lambda=1$; initial number of susceptible shrimp, $S_{0}=12$; initial number of dead infected carcasses (dose), $D_{0}=1$.

(ש) Epidemic threshold values; other symbols as in Fig. 6

doses was 1.0 (Fig. 7). Interestingly, the effect of dose on the threshold was positive, the greater the initial dose the greater the number of susceptible shrimp must be for an epidemic to follow (Table 2). An epidemic requires a peaked epidemic curve and therefore an increase in the number of infections from $t_{0}$ to $t_{1}$. Because each of the simulations was started with a successively larger number of initial infections, a correspondingly larger number of infected hosts at $t_{1}$ is required. When $D_{0}$ was 1 , more than 1 infection was necessary at $t_{1}$, when $D_{0}$ was 2 , more than 2 infections were necessary at $t_{1}$; and so forth.

Transmission coefficient $\left(\boldsymbol{\beta}_{\mathrm{d}}\right)$. Increasing $\beta_{\mathrm{d}}$ from 0.01 to 0.98 , while holding the other coefficient values as in Table 1, increased the overall speed of the epidemic in Litopenaeus vannamei because both the time to peak and the duration of the epidemic were decreased (Fig. 8). Increasing $\beta_{\mathrm{d}}$ also increased the size of the epidemic (Fig. 8). The relationship between $\beta_{d}$ and the threshold was negative (Fig. 8), because as $\beta_{d}$ increased, the rate at which acute infections develop also increased, and the threshold is determined by the relative rates of production of new infections and the removal of infectious material.
Patency coefficient (v). The consequences of increasing $v$ from 0.02 to 1.0 on the characteristics of a WSSV epidemic, with the other coefficient values as in Table 1, is displayed in Fig. 9. Increasing the patency coefficient of WSSV in Litopenaeus vannamei increased the speed of the epidemic by decreasing the time to peak and decreasing the duration of the epidemic. The final size of the epidemic at all values of $v$ was 1.0. Note that patency does not appear in Eq. (6), so it could not affect the threshold.

Virulence coefficient $\left(\boldsymbol{\alpha}_{\mathrm{a}}\right)$. Increasing $\boldsymbol{\alpha}_{\mathrm{a}}$ from 0.01 to 0.98 , while holding the other conditions constant as in Table 1, increased the overall speed of the epidemic by decreasing both the time to peak and the duration of the epidemic (Fig. 10). The final size of the epidemic at all values of $\alpha_{a}$ was 1.0. Note that virulence does not appear in Eq. (6) so it could not affect threshold.

Area coefficient $(\boldsymbol{\lambda})$. Increasing $\lambda$ from 0.1 to 9.8 , while holding the other conditions constant as in Table 1, caused an overall slowing of the epidemic in Litopenaeus vannamei, as indicated by an increase in both the time to peak and the duration of the epidemic (Fig. 11). The final size of the epidemic at all values of $\lambda$ was 1.0 . The effect of increasing $\lambda$ on the epidemic threshold was positive, because as area increases the

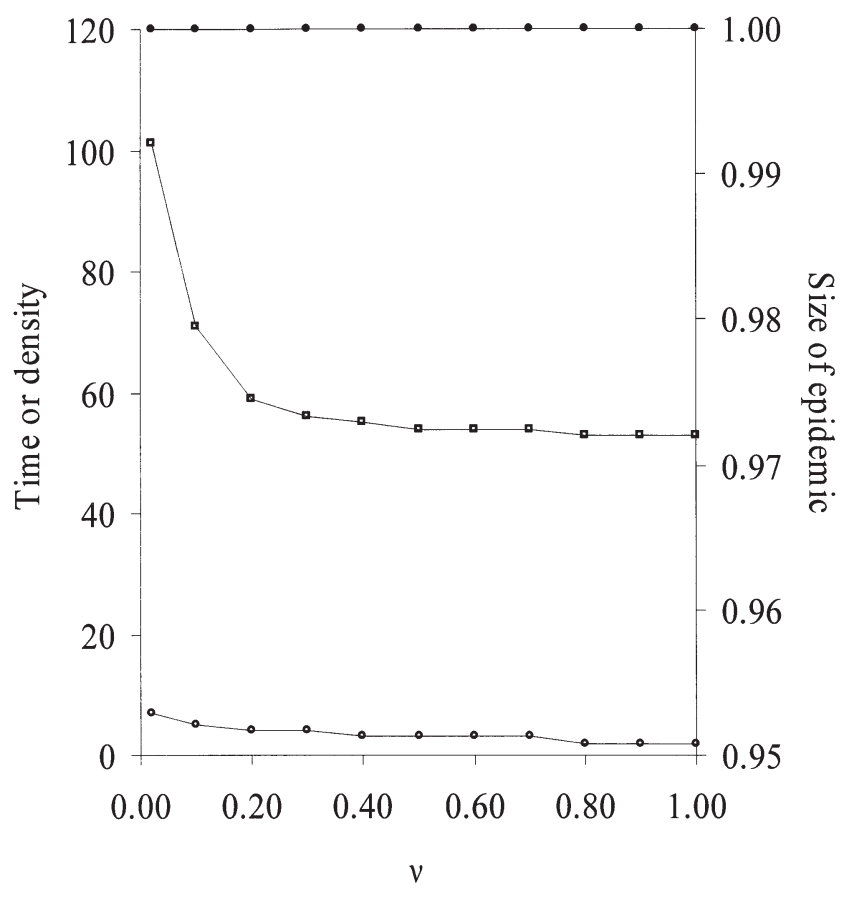

Fig. 9. Litopenaeus vannamei. Effect of patency coefficient, $v$, on characteristics of model WSSV epidemics. Transmission coefficient from a dead infected carcass, $\beta_{\mathrm{d}}=0.5$; acute virulence coefficient, $\alpha_{a}=0.3$; removal coefficient of dead infected carcasses, $\delta=0.1$; area coefficient, $\lambda=1$; initial number of susceptible shrimp, $S_{0}=12$; initial number of dead infected carcasses (dose), $D_{0}=1$. Symbols as in Fig. 6 


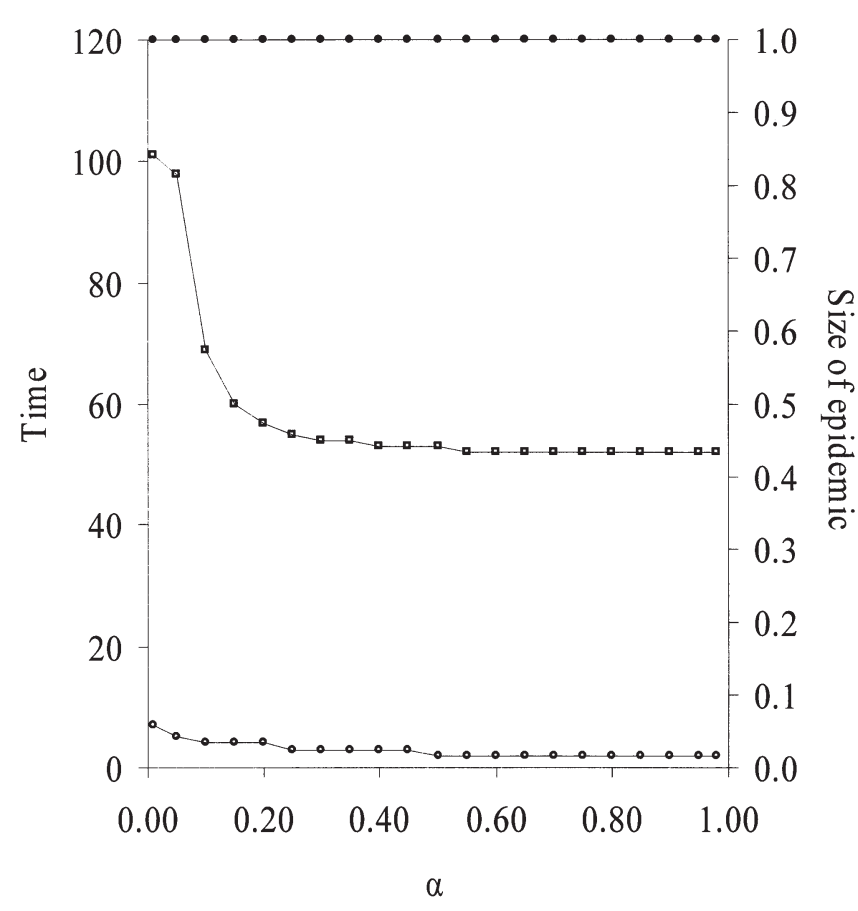

Fig. 10. Litopenaeus vannamei. Effect of acute virulence coefficient, $\alpha_{a}$, on characteristics of a model WSSV epidemic. Transmission coefficient from a dead infected carcass, $\beta_{\mathrm{d}}=$ 0.5 ; patency coefficient, $v=1$; removal coefficient of dead infected carcasses, $\delta=0.1$; area coefficient, $\lambda=1$; initial number of susceptible shrimp, $S_{0}=12$; initial number of dead infected carcasses (dose), $D_{0}=1$. Symbols as in Fig. 6

proportion of the total area that a shrimp covers in a unit of time decreases and fewer exposures, as well as transmissions, can be made per unit of time.

Removal coefficient ( $\delta$ ). Increasing $\delta$ from 0.01 to 0.98 , while holding the other conditions constant, as in Table 1, decreased the duration of the model epidemic in Litopenaeus vannamei but somewhat increased the time to peak (Fig. 12). The final size of the epidemic was decreased from 1.0 to 0.5 at high values of $\delta$ (Fig. 12). As $\delta$ increased, the threshold also increased (Fig. 12).

\section{DISCUSSION}

Ours is the first attempt to place an economically important shrimp pathogen into a mathematical framework. Epidemic models can aid the analysis and understanding of infectious disease outbreaks and may contribute to the development of disease-control strategies.

Our model contains 4 important coefficients that control the dynamics of WSSV model epidemics, the transmission coefficient, the virulence coefficient, the patency coefficient, and the removal coefficient. The significance of these coefficients suggests that they may be keys to understanding the dynamics of epidemics of WSSV in the field. However, to test their importance and to test possible management practices based on manipulating the coefficients, it is essential that the coefficients be measurable.

Fortunately, the model suggests methods for estimating the coefficients (Soto \& Lotz 2001). For example, a formula for the transmission coefficient can be obtained by solving for $\beta_{\mathrm{d}}$ in Eq. (1):

$$
\beta_{\mathrm{d}}=1-\exp \left(\frac{\ln \left(\frac{S_{1}}{S_{0}}\right)}{\frac{D_{0}}{\lambda}}\right)
$$

An estimate of the transmission coefficient can then be obtained from the initial number of susceptible and dead infected shrimp, $S_{0}$ and $D_{0}$ respectively, and the number of susceptible shrimp remaining at the end of the time period of interest, $S_{1}$ (Soto \& Lotz 2001). Experimentally, susceptible shrimp are exposed to a single dead infected shrimp in an arena of specified area for a specified period of time, then the exposed shrimp are isolated individually in order that any infections become patent, and finally all exposed shrimp are

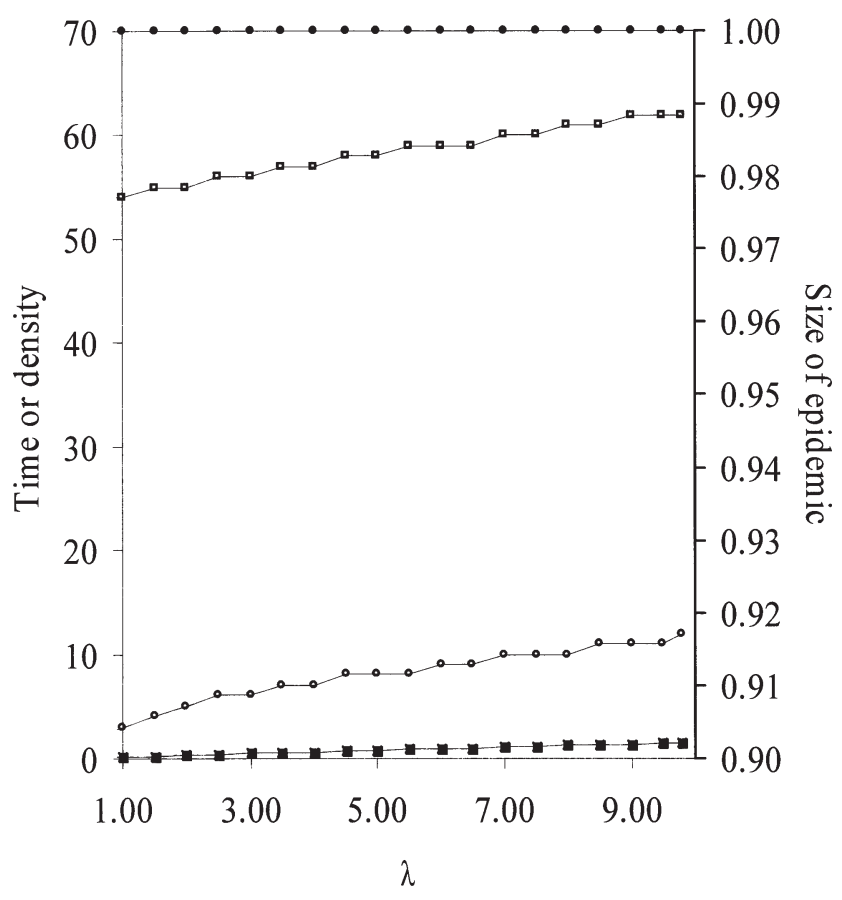

Fig. 11. Litopenaeus vannamei. Effect of area coefficient, $\lambda$, on characteristics of a model WSSV epidemic. Transmission coefficient from a dead infected carcass, $\beta_{\mathrm{d}}=0.5$; patency coefficient, $v=0.5$; acute virulence coefficient, $\alpha_{a}=0.3$; removal coefficient of dead infected carcasses, $\delta=0.1$; initial number of susceptible shrimp, $S_{0}=12$; initial number of dead infected carcasses (dose), $D_{0}=1$. (घ) Epidemic threshold values; other symbols as in Fig. 6 
examined to determine infection status (Soto \& Lotz 2001).

In addition to providing methods for empirical estimation of coefficients under various conditions, our model provides insights that help to clarify our understanding of epidemics. Consider the effect of host population size and density. The density of a host population is determined by the area that the host population occupies. Our model includes an explicit coefficient for area $(\lambda)$, the inclusion of which helps to make clear the meaning of $S_{0}$. deJong, in a series of publications (Bouma et al. 1995, deJong et al. 1995a,b), pointed out that true mass-action epidemic models (of which the Reed-Frost model is one) should be formulated explicitly in terms of density rather than in the more commonly used absolute number (pseudo-mass action). However, even when absolute numbers have been, used the actual meaning of those numbers has sometimes been interpreted as density and at other times as absolute numbers (Bouma et al. 1995).

The number of susceptible hosts in our model is the absolute number not the density; however, by including an area coefficient, density becomes part of the model. By manipulating the area coefficient or the number of susceptible individuals in the model, one can manipulate the density. In fact, by increasing the absolute number and also increasing the area coefficient accordingly one can increase the absolute size of the host population without increasing the density. In this way, one can evaluate the effect of area independently of the effect of density.

In our consideration of the area coefficient, we found that an increase in the coefficient resulted in an increase in the threshold number of susceptible shrimp needed to support an epidemic. Bailey concluded that area did not affect the threshold; however, the 2 conclusions are not contradictory. Bailey addressed area at a constant density of susceptible individuals, i.e. the total number of initial susceptible individuals $\left(S_{0}\right)$ increased as area increased. In our study of area, the initial number of susceptible individuals did not increase, and therefore as area increased density was reduced. We conclude that if only area is increased, then the model's threshold value is increased. This result implies that the threshold is a function of density, not a function of the absolute number of hosts.

Manipulation of the number of shrimp, $S_{0}$, and the area coefficient, $\lambda$, generated apparently paradoxical results for the effect of density on model WSSV epidemics. The increase in density caused by an increase in $S_{0}$ showed a tendency to slow down the WSSV model epidemic (Fig. 6). However, a decrease in density caused by an increase in area $(\lambda)$ also caused the WSSV model epidemic to slow. This paradox that both increases and decreases in density slow a WSSV model

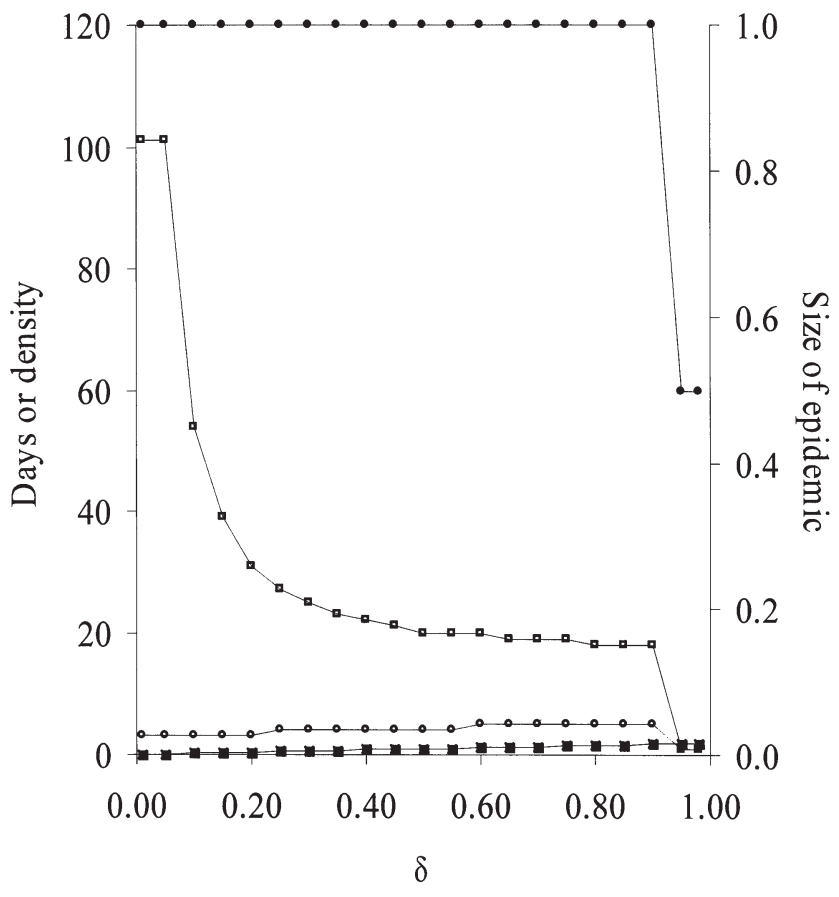

Fig. 12. Litopenaeus vannamei. Effect of dead infected carcass removal coefficient, $\delta$, on characteristics of model WSSV epidemics. Transmission coefficient from a dead infected carcass, $\beta_{\mathrm{d}}=0.5$; patency coefficient, $v=0.5$; acute virulence coefficient, $\alpha_{a}=0.3$; area coefficient, $\lambda=1$; initial number of susceptible shrimp, $S_{0}=12$; initial number of dead infected carcasses (dose), $D_{0}=1$. (घ) Epidemic threshold values; other symbols as in Fig. 6

epidemic can be explained by the fact that the 2 types of density changes are different. The change in density caused by a change in only the area coefficient maintains the absolute size of the model host population, whereas a change in density caused by a change in the number of shrimp (in a constant area) increases the number of shrimp in the host population. When there are more hosts in the population it may take longer to complete a WSSV model epidemic, even if an increase in density causes more transmission. The protraction reflects that there are more shrimp to infect than can be offset by the greater overall transmission and that the effect of density is less than the effect of population size.

Another set of factors that may help an analysis and understanding of model WSSV epidemics is disease resistance. Disease resistance is a term commonly used to describe the ability of shrimp to combat an infection, but in an epidemiological context, resistance is compounded from 3 of the epidemic coefficients identified in the model, transmission coefficient, patency coefficient, and virulence coefficient. Resistance of a shrimp would be said to increase if the likelihood of transmission were reduced, if the time for an infected shrimp to 
develop symptoms increased, or if the likelihood that mortality due to infection was reduced. If resistance is equated to the probability that an individual will not die during a WSSV epidemic, then resistance can be written as follows:

$$
P=\left(1-\beta_{\mathrm{d}}\right)+\beta_{\mathrm{d}} \cdot(1-v)+\beta_{\mathrm{d}} \cdot v \cdot\left(1-\alpha_{\mathrm{a}}\right)
$$

where $P$ is resistance, $\left(1-\beta_{\mathrm{d}}\right)$ is the probability of no transmission, $\beta_{\mathrm{d}} \cdot(1-v)$ is the probability that if transmission occurs an infected shrimp will not become patent, and $\beta_{d} \cdot v \cdot\left(1-\alpha_{a}\right)$ is the probability that a shrimp develops a patent infection but does not die. It should also be pointed out that resistance is determined by characteristics of both the host and the pathogen. A change in the characteristics of the virus could make the shrimp appear to be more resistant even though no change in the shrimp has occurred.

Each of the components of resistance has the same effect on epidemics (Figs. 8 to 10), and therefore resistance of any kind should result in a decrease in the speed at which an epidemic develops. The predicted decrease in speed implies longer pathogen presence in a pond or on a farm. Consequently, a farm using a resistant shrimp might be a source of the pathogen for a longer period of time if the farm became contaminated.

Our model identifies several factors that affect the dynamics of model WSSV epidemics. If practical ways can be developed to manipulate those factors in the field, then these methods can be tested for their utility to control actual WSSV epidemics. Among such factors are those that increase the threshold for model WSSV epidemics in Litopenaeus vannamei. Eq. (6) and Figs. 8, 11 \& 12 indicate that decreasing transmission $\beta_{\mathrm{d}}$, decreasing density (by increasing area $\lambda$ ), and increasing removal of infectious material $\delta$ will increase the threshold needed to maintain an epidemic.

As pointed out previously, decreased transmission is 1 component of pathogen resistance, and it is the only component of resistance that affects the epidemic threshold. As a consequence, it might be useful for breeding programs that select for resistance to consider selection for reduced transmissibility rather than selection for survival after infection as part of a strategy to eradicate WSSV. Although reduced mortality rate would undoubtedly lead to an economic boost for the industry, the model suggests that such a strategy would not reduce the likelihood of an epidemic occurring, even though the epidemic might be less severe. Unless reduction in the likelihood of infection rather than reduction in mortality after infection occurs, the elimination of the pathogen would not be achieved.

Decreasing the stocking density might be a useful strategy for farmers, because decreased density could bring the number (at constant area) below the threshold. Increasing the rate of decay of infected dead car- casses could also result in a higher threshold, and suggests that methods for rapid removal of dead shrimp might be worth investigating. Perhaps one possibility would be to maintain a scavenger fish in ponds that would consume dead but not live shrimp.

A final aspect of model WSSV epidemics to consider for pathogen management is the size of epidemics. Under some coefficient values, model epidemics exist that are lower than $100 \%$ (Fig. 4). Such results suggest that manipulation of factors to decrease the size of model epidemics might be worth investigating as potential controls of actual epidemics. In the present study we used coefficient estimates for WSSV obtained under a specific set of conditions, but these values did not allow clear demonstration of the effect of coefficient values on the size of the epidemic. Other conditions of estimation could result in model WSSV epidemics lower than $100 \%$. Lotz et al. (2001) explored the effect of several coefficient values on the size of a more general epidemic. In particular they found that increasing each of the components of resistance reduced the size of the epidemics. They also found that both decreasing density (number at constant area) and increasing the removal coefficient decreased the size of the epidemic. Manipulating factors that decrease the size of an epidemic might leave many susceptible shrimp unaffected by the pathogen.

In this contribution we have tried to highlight the value of a mathematical approach to the study of WSSV epidemics. It appears that this approach generates testable hypotheses for control of epidemics of WSSV. Certainly, the devastating affect that WSSV has had on shrimp aquaculture requires that no approach to virus control be overlooked.

Acknowledgements. Partial funding for this research was provided by a grant from the United States Department of Agriculture, CSREES grant number 98-38808-6019.

\section{LITERATURE CITED}

Abbey H (1952) An examination of the Reed-Frost theory of epidemics. Hum Biol 24:201-233

Bailey NTJ (1967) The simulation of stochastic epidemics in two dimensions. In: Le Cam LE, Neyman J (eds) Proceedings of the Fifth Berkley Symposium on Mathematical Statistics and Probability. Volume IV. Biology and problems of health. University of California Press, Berkeley, p 237-257

Bailey NTJ (1975) The mathematical theory of infectious disease and its applications. 2nd edn. Hafner Press, New York

Black FL, Singer B (1987) Elaboration versus simplification in refining mathematical models of infectious disease. Annu Rev Microbiol 41:677-701

Bouma A, deJong MCM, Kimman TG (1995) Transmission of pseudorabies virus within pig populations is indepen- 
dent of the size of the population. Prev Vet Med 23: 163-172

Chang PS, Lo CF, Wang YC, Kou GH (1996) Identification of white spot syndrome associated baculovirus (WSBV) target organs in the shrimp Penaeus monodon by in situ hybridization. Dis Aquat Org 27:131-39

Chou HY, Huang CY, Wang CH, Chiang HC, Lo CF (1995) Pathogenicity of a baculovirus infection causing white spot syndrome in cultured penaeid shrimp in Taiwan. Dis Aquat Org 23:165-173

Daley DJ, Gani J (1999) Epidemic modeling: an introduction. Camb Stud Math Biol 15:1-213

deJong MCM (1995a) Modelling in veterinary epidemiology: why model building is important. Prev Vet Med 25: 183-193

deJong MCM (1995b) How does transmission of infection depend on population size. In: Mollison D (ed) Epidemic models: their structure and relation to data. Cambridge University Press, Cambridge, p 84-94

Flegel TW (1997) Special topic review: major viral diseases of the black tiger prawn (Penaeus monodon) in Thailand. World J Microbiol Biotechnol 13:433-442

Flegel TW, Boonyaratpalin S, Withyachumnarnkul B (1997) Progress in research on yellow-head virus and white-spot virus in Thailand. In: Flegel TW, MacRae IH (eds) Diseases in Asian aquaculture. III. Asian Fisheries Society, Manila, p 285-295

Kermack WO, McKendrick AG (1927) A contribution to the mathematical theory of epidemics. Proc R Soc Ser A A115: 700-721

Lightner DV (1996) A handbook of shrimp pathology and diagnostic procedures for diseases of cultured penaeid shrimp. World Aquaculture Society, Baton Rouge, LA

Lightner DV, Hasson KW, White BL, Redman RM (1998) Experimental infection of western hemisphere penaeid shrimp with Asian white spot syndrome virus and Asian yellow head virus. J Aquat Anim Health 10:271-281

Lo CF, Ho CH, Peng SE, Chen CH and 7 others (1996) White spot syndrome baculovirus (WSBV) detected in cultured and captured shrimp, crabs and other arthropods. Dis Aquat Org 27:215-225

Editorial responsibility: Chris Baldock, Brisbane, Australia
Lotz JM, Davidson J, Soto MA (2001) Two approaches to epidemiology in shrimp aquaculture disease control. In: Browdy C, Jory D (eds) The new wave. Proceedings of the Special Session on Sustainable Shrimp Culture. Aquaculture 2001. The World Aquaculture Society, Baton Rouge, LA, p 155-161

Murphy FA, Fauquet CM, Bishop DHL, Ghabrial SA, Jarvis AW, Martelli GP, Mayo MA, Summers MD (1995) Virus taxonomy: The classification and nomenclature of viruses. The Sixth Report of the International Committee on Taxonomy of Viruses. Springer-Verlag, Vienna

Nakano H, Koube H, Umeawa S, Momoyama K, Hiraoka M, Inouye K, Oseko N (1994) Mass mortalities of cultured Kuruma shrimp, Penaeus japonicus, in Japan in 1993: epizootiological survey and infection trials. Fish Pathol 29: 135-139

Soto MA, Lotz JM (2001) Epidemiological parameters of white spot syndrome virus (WSSV) infections in Litopenaeus vannamei and L. setiferus. J Invertebr Pathol 78 : $9-15$

Tapay LM, Lu Y, Gose RB, Brock JA, Loh PC (1997) Infection of white-spot baculovirus-like virus (WSBV) in two species of penaeid shrimp Penaeus stylirostris (Stimpson) and $P$. vannamei (Boone). In: Flegel TW, MacRae IH (eds) Diseases in Asian aquaculture. III. Asian Fisheries Society, Manila, p 297-302

Tsai MF, Kou GH, Liu HC, Liu KF, Chang CF, Peng SE, Hsu HC, Wang CH, Lo CF (1999) Long-term presence of white spot syndrome virus (WSSV) in a cultivated shrimp population without disease outbreaks. Dis Aquat Org 38: 107-114

Wang CS, Tang KFJ, Kou GH, Chen SN (1997) Light and electron microscopic evidence of white spot disease in the giant tiger shrimp, Penaeus monodon (Fabricius), and the kuruma shrimp, Penaeus japonicus (Bate), cultured in Taiwan. J Fish Dis 20:323-331

Wongteerasupaya C, Vickers JE, Sriurairatana S, Nash GL, and 6 others (1995) A non-occluded, systemic baculovirus that occurs in cells of ectodermal and mesodermal origin and causes high mortality in the black tiger prawn, Penaeus monodon. Dis Aquat Org 21:69-77

Submitted: September 5, 2000; Accepted: January 14, 2002 Proofs received from author(s): July 9, 2002 